\title{
ADAPTIVE CONTROL OF A SOLAR FURNACE FOR MATERIAL TESTING
}

\author{
E.F. Camacho ${ }^{*, 1}$ M. Berenguel ${ }^{*, 2}$ F.J. García-Martín ${ }^{*}$ \\ and F.R. Rubio* \\ * Universidad de Sevilla. Escuela Superior de Ingenieros. \\ Departamento de Ingeniería de Sistemas y Automática. \\ Camino de Los Descubrimientos s/n, 41092, Sevilla, SPAIN. \\ Tfn: 34-5-4487347. Fax: 34-5-4487340. \\ E-mail: eduardo@cartuja.us.es
}

\begin{abstract}
This paper presents an adaptive control system for controlling the temperature of a solar furnace, which is a high solar concentrating facility made up of heliostats tracking the sun and reflecting solar radiation onto a static parabolic concentrating system at the focal spot of which a high percentage of the solar energy collected by the collector system is concentrated in a small area. A large attenuator (shutter) placed between the collector system and the concentrator serves to control the amount of solar energy used for heating the samples placed at the focal spot. The paper shows the results obtained in the application of adaptive PI controllers to a solar furnace, incorporating feedforward action, anti-windup and slew rate constraint handling mechanisms. Copyright (C) 1998 IFAC
\end{abstract}

Keywords: Adaptive PI control, solar furnace, material testing, actuator constraints

\section{INTRODUCTION}

One of the most promising fields for solar energy nowadays is that of solar furnaces for material testing and treatment. A solar furnace is a high concentrating facility made up of a collector system with tracking and a static parabolic concentrating system at the focal spot of which a high percentage of the solar energy collected by the collector system is concentrated within a small area. One attenuator (shutter) can be used between the collector system and the concentrator and another smaller, fast-reaction one between the concentrator and the focal spot to produce

\footnotetext{
1 The authors would like to thank CICYT for partially funding this work under grants TAP-96-884CP and TAP95-0370 and personnel of the Plataforma Solar de Almería, especially Diego Martínez and José Rodríguez.

$2 \mathrm{M}$. Berenguel is currently working at the Universidad de Almería (SPAIN) within the Departamento de Lenguajes y Computación (Area de Ingeniería de Sistemas y Automática)
}

thermal shock. Within the test zone a test table movable in three dimensions is placed in the area of the focal spot. An excellent description and overview of different solar furnaces can be found in (Martínez, 1996).

Tests in a solar furnace usually aim at improving the mechanical properties of different samples (steel, ceramic composites such as alumina, etc.) by means of heating the samples following many different temperature patterns. The control problem is a difficult task as:

- The dynamic characteristics of the samples are quite different depending on their nature and greatly depend on the temperature introducing a high nonlinearity, which makes the behaviour of the controlled system change with the operating conditions.

- The control specifications are severe (rate of temperature increase/decrease, step changes, 
etc.) and have to be achieved with small errors.

- The system suffers from disturbances caused by solar radiation variations, which make the reproduction of the conditions of a determined test impossible.

- Limitations exist in the maximum temperature achievable by the materials and different constraints in the actuator (amplitude, slew rate, etc.).

The paper shows the results obtained in the application of adaptive PI controllers to a solar furnace, incorporating feedforward action, antiwindup and slew rate constraint handling mechanisms. The paper is organized as follows: a brief description of the solar furnace and the dynamic models of the system for control purposes is given in Section 2. Section 3 is devoted to explain the development and implementation of feedforward and feedback control schemes to be used within an adaptive control framework. Section 4 presents some experimental results and finally, some conclusions are given in Section 5 .

\section{DESCRIPTION AND MODELS OF THE SYSTEM}

The Plataforma Solar de Almería (PSA) Solar Furnace is mainly devoted to material treatment and many research institutions have carried out various test campaigns on metallic and ceramic samples (Rodríguez et al., 1996). It consists of:

- Four heliostats which reflect the sun light onto the concentrator disk, each having a reflecting area of $53.61 \mathrm{~m}^{2}$, computer controlled sun tracking mechanism and $90 \%$ reflectivity.

- A computer controlled louvered shutter formed by 30 panels which regulates the incoming light onto the concentrator (energy flux entering the furnace), with a total dimension of $11.5 \mathrm{~m} \times 11.2 \mathrm{~m}$ and 15896 positions between $0^{\circ}$ (open) and $55^{\circ}$ (closed).

- A collector which concentrates the incoming sun-light onto a test table consisting of 89 spherical facets $(0.91 \mathrm{~m} \times 1.21 \mathrm{~m})$ with a total reflecting area of $98.5 \mathrm{~m}^{2}$, reflectivity $94 \%$, concentration (peak) $3000 \mathrm{MW} / \mathrm{m}^{2}$, peak power $60 \mathrm{KW}$ and focus size $22 \mathrm{~cm}$.

- A test table controlled by a computer where the samples are placed, mobile on 3 axis.

Fig. 1 shows an outer view of the PSA solar furnace. It can be seen how four heliostats concentrate solar radiation onto the shutter which controls the fraction of beam radiation which goes into the furnace for material testing purposes. Fig. 2 shows how the solar furnace operates inside. The fraction of the solar radiation collected by

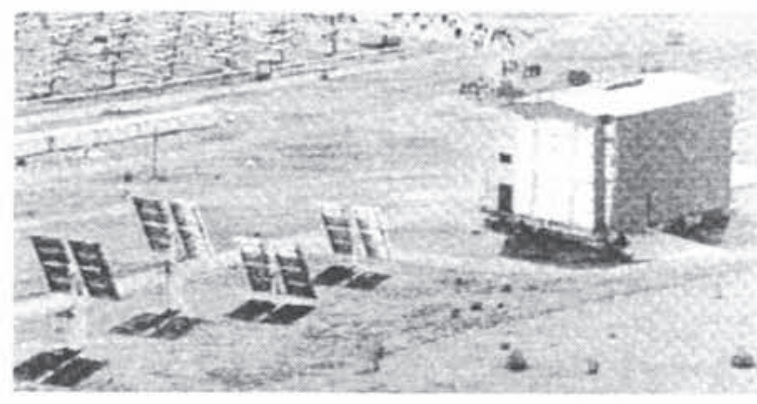

Fig. 1. Outer view of the PSA solar furnace

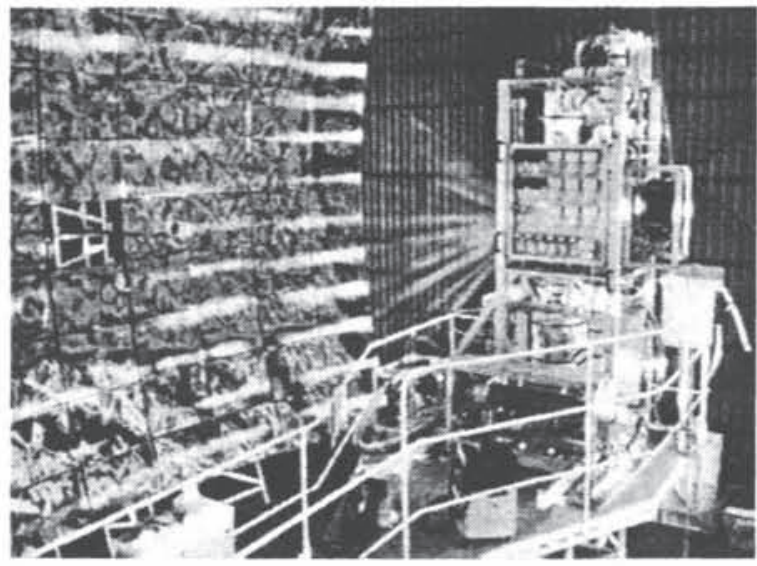

Fig. 2. Inner view of the PSA solar furnace

the heliostats incises onto a parabolic receiver to concentrate the radiation the focal point where the sample is placed.

The heliostat movement is calculated and controlled by a computer using the geographic latitude and longitude, the sun trajectory, the mirror characteristics, etc. in an open loop fashion. This paper introduces a scheme to control the temperature profile of the samples by the adjustment of the shutter position in spite of the variability of the input energy provided by the sun (due to its daily cycle and the presence of passing clouds) and the different temperature profiles needed to perform the tests.

From the control viewpoint, the input signal to the system is the aperture of the shutter and the output signal is the temperature measured by a thermocouple placed over or behind the sample. A simplified model of the system was obtained from first principles, which simplified energy balance is introduced. For the sake of clarity, the dependence on time of different variables has not been explicitly written.

\section{Input thermal power-}

The heliostats reflect a fraction $I_{s}=\rho_{h} I$ of the direct solar radiation $I$ coming from the sun ( $\rho_{h}$ being the mirror reflectivity). The shutter aperture limits the reflected solar radiation which goes through it. The shutter opening is performed 
by using an AC motor which rotates the axle to which the panels are linked between $0^{\circ}$ (fully open) and $55^{\circ}$ (fully closed). As can be seen in Fig. 3 the relationship between the aperture angle $(\alpha)$ and the aperture $(A)$ is $A=L\left(\sin \alpha_{0}-\sin \alpha\right)$, where $L$ is the length of the panel, $\alpha_{0}$ is the angle in which the shutter is completely closed (zero aperture) and $\alpha$ is the angle which indicates the aperture percentage. As the encoders are such that the control signal to the motor which moves the shutter is the percentage of rotated angle $(U)$ with respect to $\alpha_{0}$, that is, $\alpha=(1-U / 100) \alpha_{0}$, the aperture $A$ related to the control signal $U$ is given by the nonlinear relationship $A=1-(\sin [(1-$ $\left.\left.U / 100) \alpha_{0}\right] / \sin \alpha_{0}\right)$.
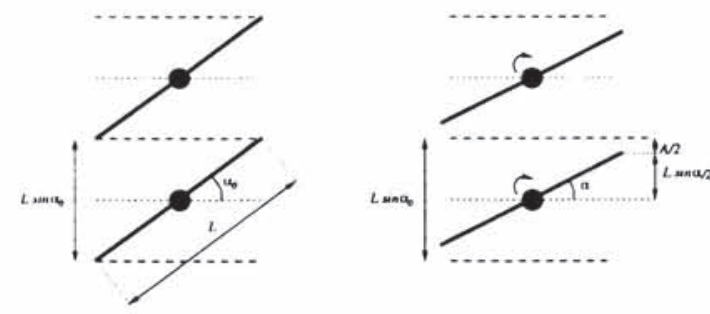

Fig. 3. Shutter aperture

The power density behind the shutter is: $I_{c}=$ $I_{s} A=I \rho_{h}\left[1-\left(\sin \left[(1-U / 100) \alpha_{0}\right] / \sin \alpha_{0}\right)\right]$. This power density is collected by the concentrator proportionally to its surface $S_{c}$ and is projected towards the focus with different losses due to its reflectivity $\rho_{c}$. The power obtained at the concentrator output is: $P=I_{c} S_{c} \rho_{c}=I \rho_{h} \rho_{c} S_{c}[1-$ $\left.\left(\sin \left[(1-U / 100) \alpha_{0}\right] / \sin \alpha_{0}\right)\right]$. The energy received by the sample depends upon its surface $\left(S_{s}\right)$ due to the flux distribution at the focus of the system. Supposing that the energy flux is uniformly distributed within the focus (approximation), the input power is:

$$
\begin{aligned}
P_{i} & =\frac{\rho_{h} \rho_{c} S_{c}}{S_{f(90 \%)} \sin \alpha_{0}} I S_{s} \alpha_{a}\left[\sin \alpha_{0}-\right. \\
& \left.-\sin \left[(1-U / 100) \alpha_{0}\right]\right]
\end{aligned}
$$

$S_{f(90 \%)}$ and $\alpha_{a}$ being the focus area (90\% of the power is concentrated within a circumference with a diameter of about $20 \mathrm{~cm}$ ) and absorption capacity of the sample respectively.

\section{Radiation and convection thermal losses-}

The radiation losses are given by $P_{r}=\alpha_{e} \sigma S_{s}\left(T^{4}-\right.$ $T_{a}^{4}$ ) where $\sigma$ is the Stephan-Boltzmann constant, $\alpha_{e}$ the emissivity of the sample (determines the capacity to irradiate energy), $T_{a}$ is the environmental temperature and $T$ is the temperature (supposedly uniform) of the sample given in Kelvin degrees.
The power lost by convection mechanisms can be approximated by $P_{c}=\alpha_{c} S_{s}\left(T-T_{a}\right)$, where constant $\alpha_{c}$ indicates the capacity of the sample to interchange heat with the air depending on the position of the sample and on the properties of the air.

\section{Energy balance-}

The energy balance is given by: $P_{i}-P_{r}-P_{c}=$ $d E / d t$, where $E$ is the thermal energy of the sample $\left(E=m C_{e} T, m\right.$ being the mass of the sample, $C_{e}$ the specific heat and $T$ the temperature in Kelvin degrees). By substituting each one of the terms:

$$
\begin{aligned}
& \frac{\rho_{h} \rho_{c} S_{c}}{S_{f(90 \%)} \sin \alpha_{0}} I S_{s} \alpha_{a}\left[\sin \alpha_{0}-\sin \left[(1-U / 100) \alpha_{0}\right]\right] \\
& -\alpha_{e} \sigma S_{s}\left(T^{4}-T_{a}^{4}\right)-\alpha_{c} S_{s}\left(T-T_{a}\right)=\frac{d\left(m C_{e} T\right)}{d t}
\end{aligned}
$$

At an equilibrium point $\left(U_{0}, T_{0}\right)$ the energy variation in time is null. If a small perturbation around the equilibrium point is produced $\left(U=U_{0}+\right.$ $u$ and $T=T_{0}+\zeta$ ), a linearized model of the system which reproduces the behaviour of the system around a determined operating point can be obtained. By linearizing both the sinusoidal term and all those terms with high powers, the following relationship can be obtained:

$$
\begin{aligned}
m C_{e} \frac{d \zeta}{d t} & =K_{1} I S_{s} \alpha_{a} \cos \left[\left(1-U_{0} / 100\right) \alpha_{0}\right] u- \\
& -4 \alpha_{e} \sigma S_{s} T_{0}^{3} \zeta-\alpha_{c} S_{s} \zeta
\end{aligned}
$$

where constant

$$
K_{1}=\left(\rho_{h} \rho_{c} S_{c} \alpha_{0}\right) /\left(100 S_{f(90 \%)} \sin \alpha_{0}\right)
$$

( 24.95 for this system) is constituted of characteristic parameters of the system, independently of the type of material. If null initial conditions are supposed and the Laplace Transform is applied to this linearized model $(u(t) \rightarrow U(s) ; \zeta(t) \rightarrow T(s))$, a first order transfer function can be obtained $T(s) / U(s)=K /(1+\tau s)$, where:

$$
K=\frac{K_{1} \alpha_{a} I \cos \left[\left(1-U_{0} / 100\right) \alpha_{0}\right]}{4 \sigma \alpha_{e} T_{0}^{3}+\alpha_{c}}
$$

and

$$
\tau=\frac{m C_{e}}{S_{s}\left(4 \sigma \alpha_{e} T_{0}^{3}+\alpha_{c}\right)}
$$


From this simplified model, some simple but important conclusions can be drawn:

- The gain of the system depends proportionally on the solar radiation, and the linearized system time constant is independent of it.

- The variation in the gain of the system with the cosine of a term involving the initial aperture angle and the equilibrium operating point aperture models the shutter nonlinearity so that, the higher the aperture of the shutter, the higher the system gain.

- Both the system gain and the time constant inversely depend on the cube of the temperature and decrease as the capacity of the material to loose energy augments (large emissivity and convection loss rate).

- The gain is independent of the sample area, but the time constant of the system diminishes as the area of the sample augments.

- Those samples with high specific heat lead to an increase in the time constant of the system.

Notice that all these conclusions are valid under the assumptions made to obtain the linearized model. The shutter actuation mechanism introduces two nonlinearities: one of saturation type ( $0 \%-100 \%$ aperture) and the other of slew rate type, as the aperture rate response of the shutter is fixed at $5 \%$ each second, lasting 20 seconds opening from $0 \%$ to $100 \%$.

\section{FEEDFORWARD AND FEEDBACK CONTROLLERS}

Many different temperature profiles are required for a wide class of tests (Rodríguez et al., 1996) covering melting experiences, production of a super alloy wear resistant coating for high temperature applications, etc. The specifications for the operation can be translated into classical control ones:

- The steady state error for a ramp input should be as small as possible, as temperature profiles of this type (or more complex ones) are frequently requested.

- A non-oscillatory response for the closed loop system is desirable, as sometimes is necessary to work at temperatures close to the melting point.

- A settling time after a step in the reference temperature of less than 1-3 minutes is desirable, although this is not a critical parameter in this type of tests.

A sampling time of 3 seconds has been used for control purposes, and relevant signals are stored each second by a Data Acquisition System.

\subsection{Feedforward control}

As has been demonstrated in other types of solar plants (Camacho et al., 1992; Camacho et al., 1997) the use of feedforward controllers if fundamental for systems subjected to measurable disturbances. These feedforward controllers can be used in classical feedback loops as a complementary control action to reject disturbances acting on the system. Two types of configurations have commonly been used for solar processes: parallel and series, which main advantages and drawbacks are discussed in (Camacho et al., 1992; Camacho et al., 1997).

The most important disturbance acting on the system is the direct solar radiation, which measurement can be used to cancel, or at least diminish, the effect that changes in this variable produce in the value of the temperature of the sample. The continuous system transfer function can be written as: $G(s)=K^{\prime} I /(1+\tau s)$, where the solar radiation proportionally affects the linearized system gain. In order to achieve control results independent of the value of the solar radiation, if a block with a transfer function $G_{f f}=I_{\text {ref }} / I$ is placed in the forward path (series feedforward controller), the resulting global transfer function is theoretically "independent" of the solar radiation values, having a gain constant (if the supposition of the operation around an operating point is fulfilled) corresponding to a solar radiation level (input energy) equal to $I_{r e f}$, which has been taken close to the mean value of $900 \mathrm{~W} / \mathrm{m}^{2}$. This is an approximation valid both for steady state and transient conditions, as the transfer function which relates the changes in the sample temperature to changes in solar radiation has theoretically the same characteristic polynomial as the system transfer function (when considering linear operation and without nonlinearities introduced by the actuator). This is due to the fact that a decrease in the solar radiation level has the same effect on the output temperature as a diminish in the shutter aperture.

\subsection{PI control with constraint handling}

As the plant can be approximated as a first order system and taking into account the system specifications, the decision was taken to tune and implement a PI controller. The usual PI transfer function is given by $G_{P I}(s)=K_{p}\left[1+1 /\left(T_{i} s\right)\right]$. As has been mentioned, the actuator has amplitude constraints which have been accounted for by including a classical anti-windup mechanism (Åström and Hägglund, 1996).

In many control problems, the existence of an actuator constraint of the slew rate type is not 
accounted for during the design step and the obtained controller is detuned when implemented to obtain a generally robust and well-damped performance. As the system is modelled as a first order one, a common solution in the control of these kinds of systems to obtain fast responses from simple root locus analysis is to use low integral times and high gain controllers. When implementation issues are taken into account, this is not always an adequate solution, as the output of the controller must be realistic and take the constraints into account.

Designing a PI controller for a first order system without taking into account the actuator constraints is a simple task. From the root locus analysis viewpoint and supposing the characteristic time constant of the controlled system to be at a determined location, two cases can be considered, depending on the position of the PI controller zero (depending on the value of $T_{i}$ ).

If $T_{i} \leq \tau$, different types of responses can be obtained depending on the position of the controller zero. As a trade-off design, the controller was designed requesting the existence of two real poles (although the controller zero is closer to the origin than closed loop poles so that the output of the closed loop response can surpass the reference after a set point change) so that the following condition can be obtained:

$$
T_{i} \geq 4 \tau K K_{p} /\left(K K_{p}+1\right)^{2} \text { with } T_{i} \leq \tau
$$

An approach is adopted to express the limitations in the design of the controller imposed by slew rate nonlinearities so that the PI can be designed to incorporate low-pass action (limited bandwidth) according to the limited rate of variation in the shutter aperture in such a way that all frequencies higher than a determined cut-off frequency $w_{0}$ are attenuated at least in $20 \mathrm{~dB}$. The worst case input signal for the actuator is one sinusoidal signal with amplitude equal to 100 and with a maximum slope equal to 5 . So, if a sinusoidal signal $100 \sin \left(w_{o} t\right)$ is introduced in the actuator input, the derivative of this signal in $t=0$ gives the maximum rate variation which has to be limited to 5 , leading to a value $w_{0}=1 / 20 \mathrm{rad} / \mathrm{s}$. This approximation introduces another criterion for the PI design. The analytical expression of the module of the frequency response of a PI controller is given by $|C(j w)|=K_{p} /\left(T_{i} w\right) \sqrt{T_{i}^{2} w^{2}+1}$. For $w=w_{0}$, the gain of the PI controller must be less or equal to $-20 \mathrm{~dB}$. By performing a few operations, another relationship between $T_{i}$ and $K_{p}$ can be obtained:

$$
T_{i} \geq 20 K_{p} / \sqrt{0.01-K_{p}^{2}}
$$

In this way, two inequalities (2) and (3) relating $T_{i}$ and $K_{p}$ are obtained. The set of possible PI parameters which fulfill both inequalities is represented in Fig. 4. The upper curve represents equation (2), that is, the curve delimits the region over which the closed loop poles are real poles. The lower curve is given by equation (3) and represents the limitation in the controller bandwidth to adjust the controller dynamics to the shutter constraints. The region within which the controller parameters must be situated (shadowed in Fig. 4) is below the line $T_{i}=\tau$ and over the lines representing equations (2) and (3). The point selected for design purposes is given by the intersection of these last curves, leading to the minimum integral time within the possible range. The parameters corresponding to this point can be found by solving the equality given by equations (2) and (3). Both iterative methods or approximations can be used to solve it. If an analytical solution is convenient to be used in adaptive control schemes, an approximated upper bound on the curve representing equation (3) can be found in such a way that the solution is given by a quadratic function. It can be demonstrated (García-Martín et al., 1996) that an upper bound of the function on the right side of equation (3) is given by $2 K_{p} /\left(0.01-K_{p}^{2}\right)$, that is, $T_{i} \geq 2 K_{p} /\left(0.01-K_{p}^{2}\right)$ fulfills equation (3). In this way, the controller parameters can be found by solving the equality:

$$
\frac{4 \tau K K_{p}}{\left(K K_{p}+1\right)^{2}}=\frac{2 K_{p}}{0.01-K_{p}^{2}}
$$

which leads to the solution:

$$
K_{p}=\frac{\sqrt{K \tau\left(0.02 K^{2}+0.04 K \tau-2\right)}-K}{K(K+2 \tau)}
$$

and

$$
T_{i}=\frac{4 \tau K K_{p}}{\left(K K_{p}+1\right)^{2}}
$$

Different simulations were performed following this design method, providing similar results in cases considering both ideal and constrained actuators (García-Martín et al., 1996).

The second possibility given by root locus analysis introduces the controller zero between the origin and the system pole $\left(T_{i} \geq \tau\right)$. From the actuator constraint viewpoint, the same considerations made in previous paragraphs can be made here, and controller parameters can easily be found (García-Martín et al., 1996). Nevertheless, this second approach is more sensitive to modelling errors, as changes in system gain lead to pronounced changes in settling time.

The previous laws (4) have been used within an adaptive control scheme, as seems to be a possible solution to solve the problem of solar plants 


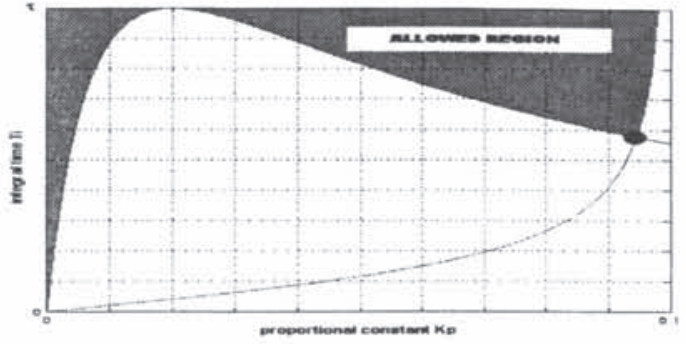

Fig. 4. $T_{i}$ vs. $K_{p}$

with different types of dynamics (Camacho et al., 1997). The control scheme implemented (Fig. 5) consists of a self-tuning controller ( $\AA$ ström and Wittenmark, 1989) in which the parameters of a simplified first order model of the plant are identified on-line (the parametric identification algorithm used is the least squares one with fixed forgetting factor). From these parameters, the controller parameters are changed according to an adaptation mechanism that relates controller parameter changes to system parameter changes (in this case given by equations (4). The adaptive controller also incorporates feedforward action, anti-windup mechanism, slew rate actuator constraints handling and identifier data prefiltering.

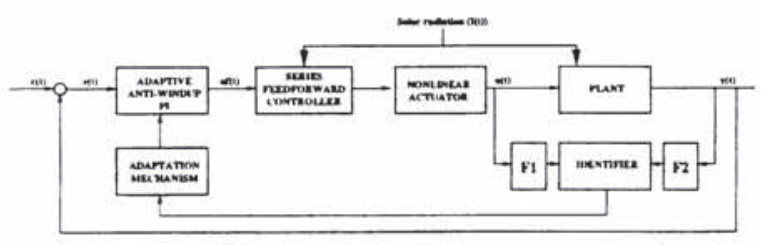

Fig. 5. Adaptive control scheme

Different problems arise when using adaptive control in solar plants (Camacho et al., 1997), the main ones being the poor system excitation (as the system identification is performed in a closed loop configuration) and the impossibility of assuring two time scales (one for the system dynamics and the other for the identified parameter dynamics). To account for these problems, different variations to classical adaptive control schemes have been introduced. The use of the series feedforward controller helps to linearize the behaviour of the system when included as a part of the estimated plant in an adaptive control scheme (Camacho $e t$ al., 1992; Camacho et al., 1997). Nevertheless, in this case, due to the slew rate constraints, problems can arise in the estimation algorithm as the input signal to the identifier may not correspond to the real input to the system. To cope with this problem, the input signal to the identifier loop is the real plant input $u(t)$ and different filters can be introduced in the identification loop (filters F1 and F2 in Fig. 5). The different possibilities are to make $\mathbf{F} 1=1$ and to multiply the output signal of the plant by the same filter used for feedfor- ward control before entering the identifier or to make $\mathbf{F} 2=1$ and to multiply $u(t)$ by the inverse of the feedforward filter. Notice that the feedforward action can also include the inverse of the nonlinearity introduced by the conversion from aperture angle to aperture percentage represented in Fig. 3.

Finally, three basic supervisory mechanisms have been implemented: limitation of the maximum and minimum values of the estimated parameters, adequate filtering of the obtained estimated system parameters (to assure the existence of two time scales) and stopping of the identifier in cases of pooer dynamic information entering the identifier.

\section{EXPERIMENTAL RESULTS}

Several tests have been performed by using the adaptive control algorithm for two kinds of materials: one sample of A316-L steel (high gains and small time constants) and another made of white zirconia (low gains and high time constants). In both cases the characteristics of the materials change with temperature. These types of samples possibly represent two extreme cases with respect to gain and time constant characteristics.

Fig. 6 represents a test with the white zirconia sample in which both the evolution of the main variables of the test (temperature, direct solar radiation and shutter aperture) and the evolution of the estimated parameters of a first order model of the plant are shown.

Filtering the estimated parameters avoids the induction of sudden changes in the controller parameters after a step change in the reference temperature. The filtered estimated values converge to adequate values after a transient which depends on the filter used, as can be seen in Fig. 6 in which the initial values of the parameters considerably differ from the real ones. In the same figure it can be seen that the trend of the estimated parameter approaches that expected of the dynamical study of the plant, as both the gain and fundamental time constant of the system decrease as the temperature of the sample augments and augment as the temperature decreases. Undesirable initial transients due to a wrong initial estimation of parameters can be avoided by using a conservative fixed PI controller during the transient in which the estimated parameters approximate the appropriate ranges or by introducing some kind of auto-tuning capability. As the estimated parameters achieve the adequate values, the overshoot obtained after a step response decreases $(34 \%$ at $500^{\circ} \mathrm{C}$ and between $13 \%$ and $24 \%$ in the rest of the test). The rise times are between 31 and 40 seconds, which constitutes quite good behaviour. 

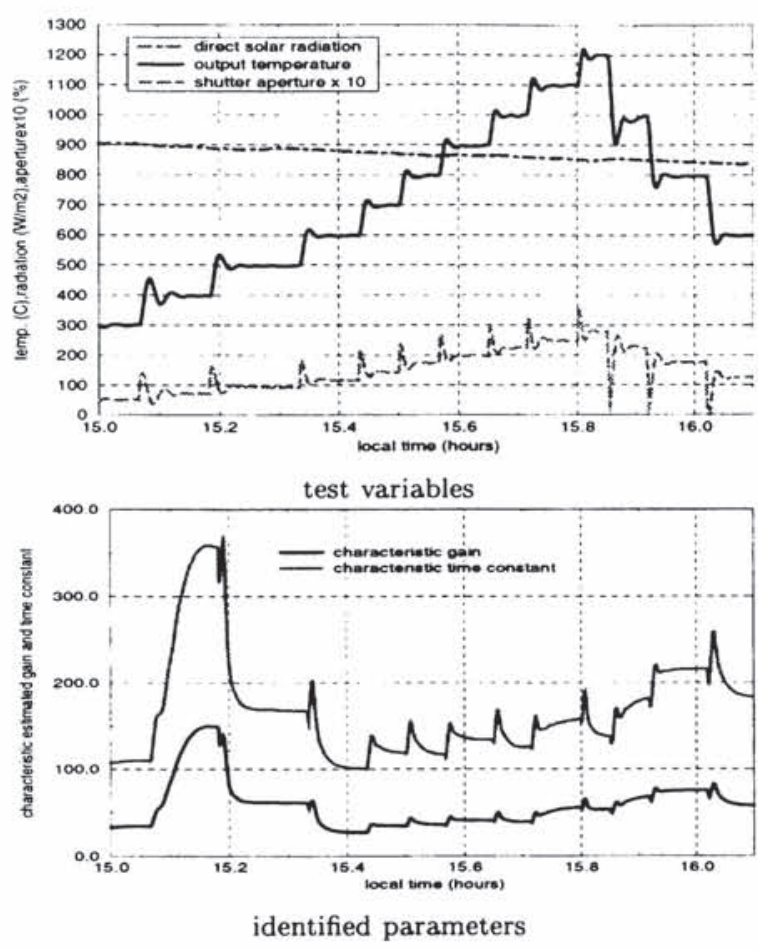

Fig. 6. Test with the adaptive PI controller $(26 / 02 / 97)$

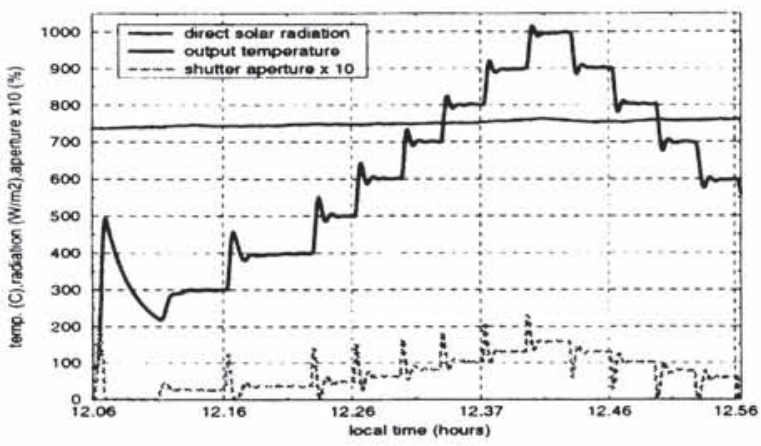

Fig. 7. Test with the adaptive PI controller $(24 / 02 / 97)$

Similar tests have been performed with an A316L steel sample, obtaining adequate results which lead to the same considerations made above. As an example, Fig. 7 shows the results obtained on February $24^{\text {th }}$ 1997. After an initial transient during which identifier excursions occur due to the wrong selection of the initial values of the selected model, the controlled system performance improves as new dynamical information enters the identifier.

A test performed on February $25^{\text {th }} 1997$ is shown in Fig. 8 with the same type of sample. The relevance of this test comes from the fact that the solar radiation conditions during this test were such that manual operation under these circumstances was impossible, even for a skilled operator. The good characteristics of set point tracking and disturbance rejection are in part due to the series feedforward action, which compensates for

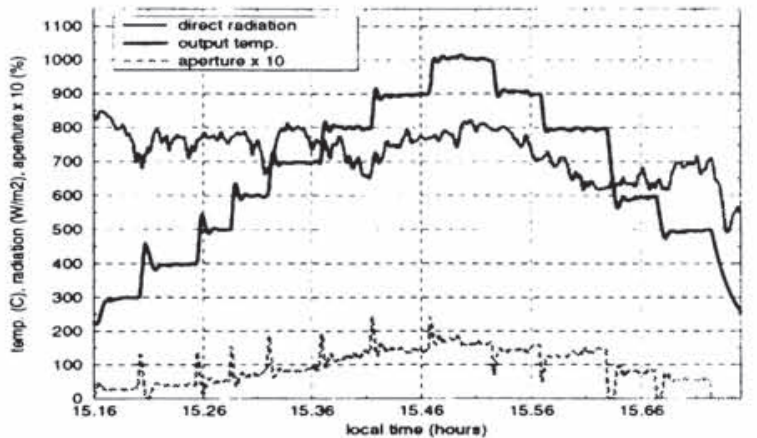

Fig. 8. Test with the adaptive PI controller $(25 / 02 / 97)$

changes in solar radiation within a band of 200 $\mathrm{W} / \mathrm{m}^{2}$ allowing the output temperature to be maintained within a band of $10^{\circ} \mathrm{C}$ around the reference.

\section{CONCLUSIONS}

An application of an adaptive PI control strategy to a solar plant for material treatment has been shown. The control scheme presented incorporates feedforward compensation, an anti-windup mechanism and actuator slew rate constraints handling in a self-tuning configuration. Different plant results have been shown when testing samples of different materials under very different temperature profiles.

\section{REFERENCES}

Åström, K.J. and B. Wittenmark (1989). Adaptive Control. Addison-Wesley.

Åström, K.J. and T. Hägglund (1996). Pid control. The Control Handbook pp. 198-209.

Camacho, E.F., F.R. Rubio and F.M. Hughes (1992). Self-tuning control of a solar power plant with a distributed collector field. IEEE Control Systems pp. 72-28.

Camacho, E.F., M. Berenguel and F.R. Rubio (1997). Advanced Control of Solar Plants. Springer Verlag. London.

García-Martín, F.J., M. Berenguel, E.F. Camacho and F.R. Rubio (1996). Automatic control of a solar furnace.

Martínez, D. (1996). Solar furnace technologies. Solar Thermal Test Facilities.

Rodríguez, J., D. Martínez and V. Shcheglov (1996). Materials treatments at the solar furnace of plataforma solar de almería under eu's dgxii hcm programme. Proc. 8th Int. Symp. on Solar Thermal Concentrating Technologies 2, 1592-1608. 